\title{
Silicon Strip Detectors for ATLAS at the HL-LHC Upgrade
}

\author{
K. Hara (U. of Tsukuba) and Y. Ikegami (KEK), for the ATLAS Upgrade Silicon Strip Community
}

The present ATLAS silicon strip (SCT) and transition radiation (TRT) trackers will be replaced with new silicon strip detectors, as part of the Inner Tracker System (ITK), for the Phase-Il upgrade of the Large Hadron Collider, HL-LHC. We have carried out intensive R\&D programs based on $n^{+}-$on-p microstrip

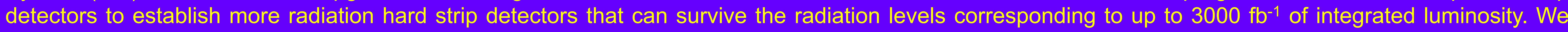
describe the main specifications for this year's sensor fabrication, followed by a description of possible module integration schema.

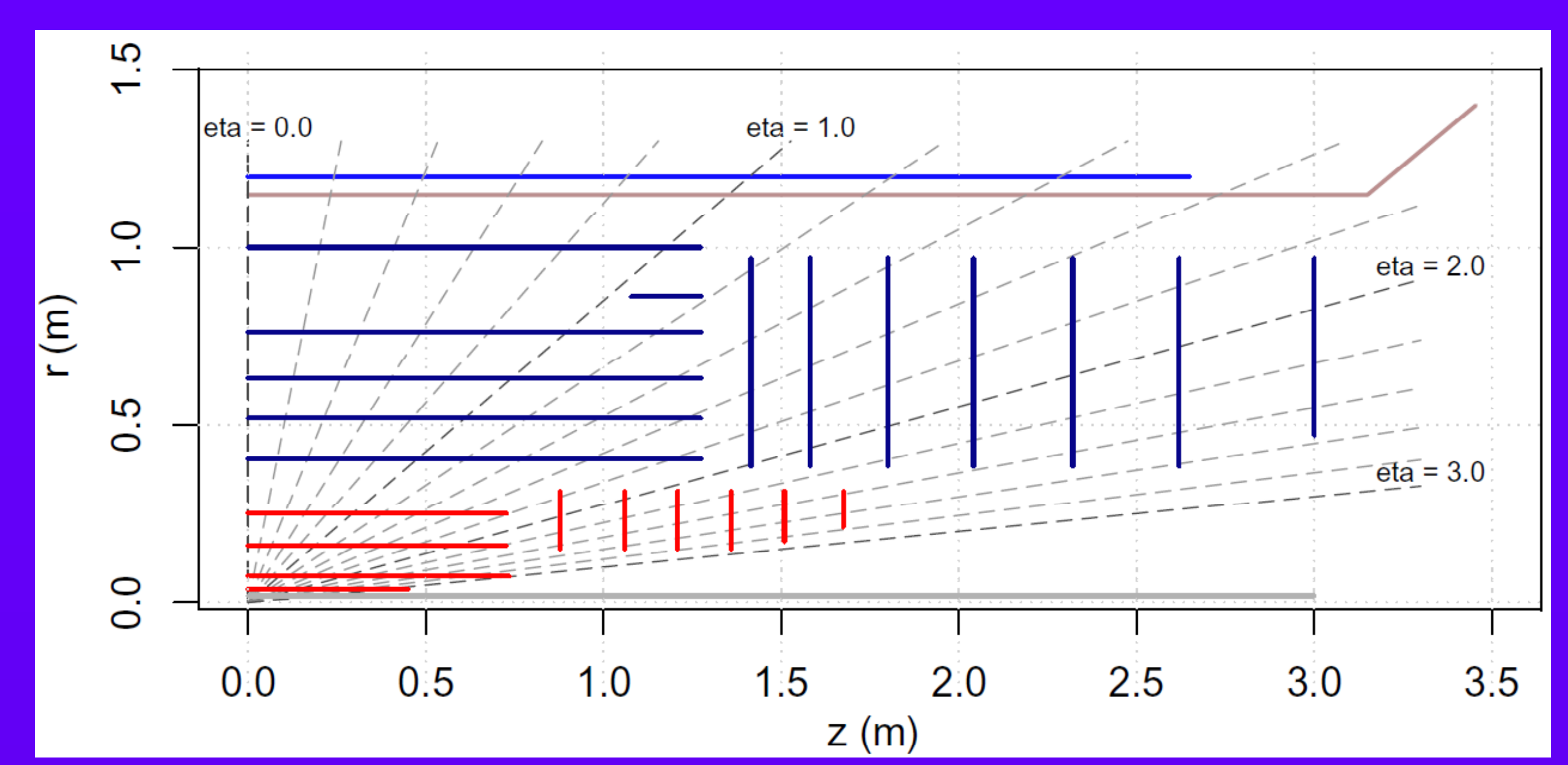

Baseline layout of the new ATLAS inner tracker for HL-LHC (pixels
strips in navy blue). The layout aims to have at least 14 silicon hits

everywhere to perform robust tracking (Lol layout).
To cope with an increase of five times in luminosity to $5 \times 10^{34} \mathrm{~cm}^{-2} \mathrm{~s}^{-1}$, the barrel strips at three radii close to the interaction region are $2.4 \mathrm{~cm}$ long while the other layers are $4.8 \mathrm{~cm}$ (current SCT strips are $12 \mathrm{~cm}$ long), both at $74.5 \mu \mathrm{m}$ pitch (ditto $80 \mu \mathrm{m}$ ). The expected fluence during their lifetime is

Short Strips: up to $1.2 \times 10^{15} 1-\mathrm{MeV} \mathrm{n}_{\mathrm{eq}} / \mathrm{cm}^{2}$

Long Strips: up to $0.56 \times 10^{15} 1-\mathrm{MeV}_{\mathrm{eq}} / \mathrm{cm}^{2}$

lonizing dose: up to $35 \mathrm{kGy}$

With the baseline layout, the barrel and end-cap strip detectors have respectively 122 and $70 \mathrm{~m}^{2}$ area, and 47M and $27 \mathrm{M}$ readout channels.

By placing outer radii strips at large radius, the resolution is expected to improve over the current Inner Detector.

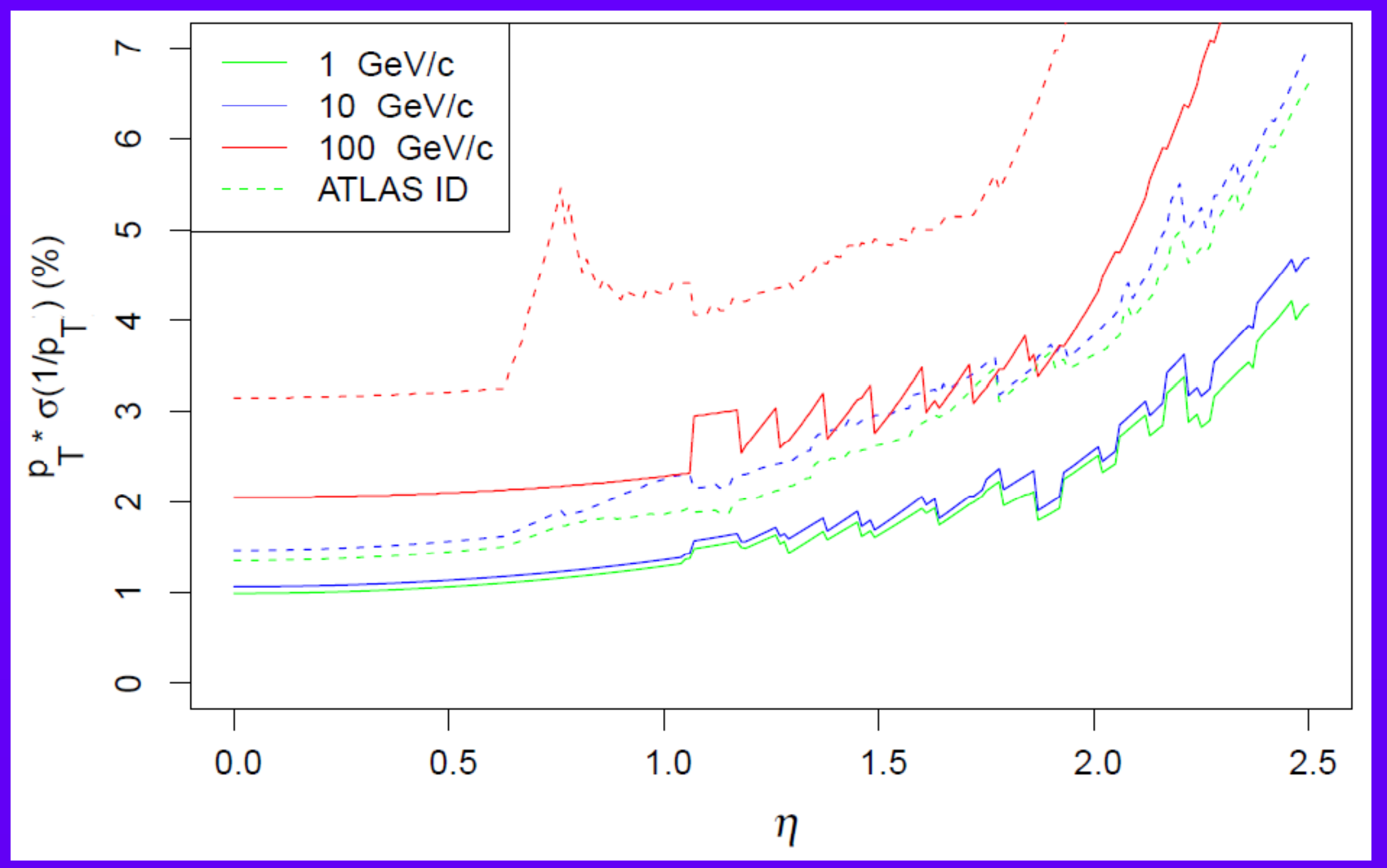

Estimated $p_{T}$ resolution for different momenta. Dashed curves are for the present ATLAS. This is an analytical calculation.
The main specifications for this year's R\&D sensor (ATLAS12) are summarized below:

- The baseline is $p$-type FZ wafers of $>4 \mathrm{k} \Omega \mathrm{cm}$ resistivity to realize initial full depletion of $<300 \mathrm{~V}$ for $320 \mu \mathrm{m}$ thickness. The strip isolation is achieved by common $\mathrm{p}$-stop structure with doping concentration of approximately $4 \times 10^{12}$ ions $/ \mathrm{cm}^{2}$

The maximum operation voltage is set at $600 \mathrm{~V}$, suitable for $500 \mathrm{~V}$ rating of the existing ATLAS cables. No microdischarge should occur below $600 \mathrm{~V}$. However the sensors will be tested up to $1 \mathrm{kV}$ to investigate stable operation at higher biases.

The interstrip capacitance to the nearest neighbor on both sides should be $0.8 \mathrm{pF} / \mathrm{cm}$ at $300 \mathrm{~V}$ bias and $\mathrm{f}_{\mathrm{TEST}}=100 \mathrm{kHz}$ $>98 \%$ of good strips are required

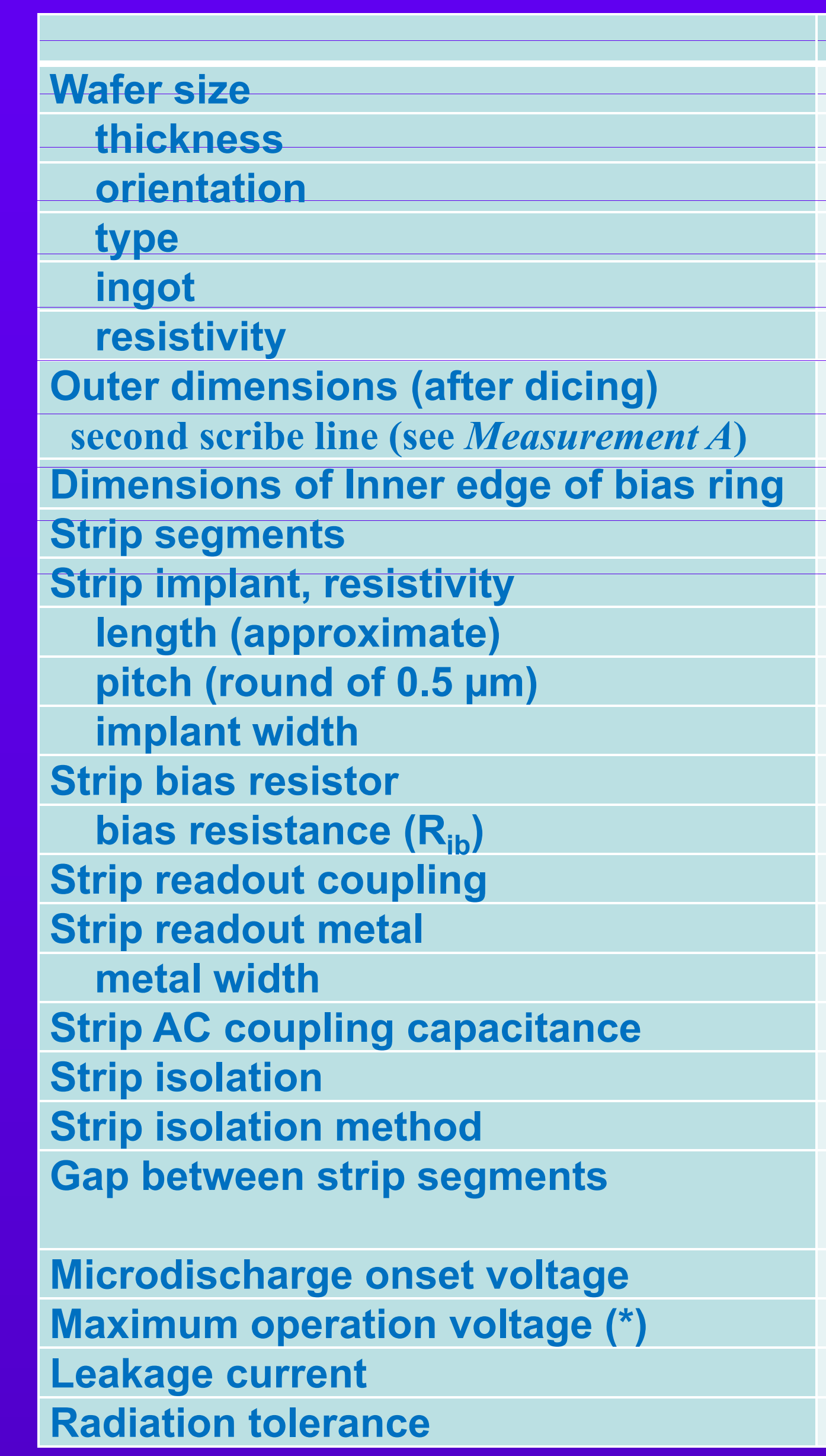

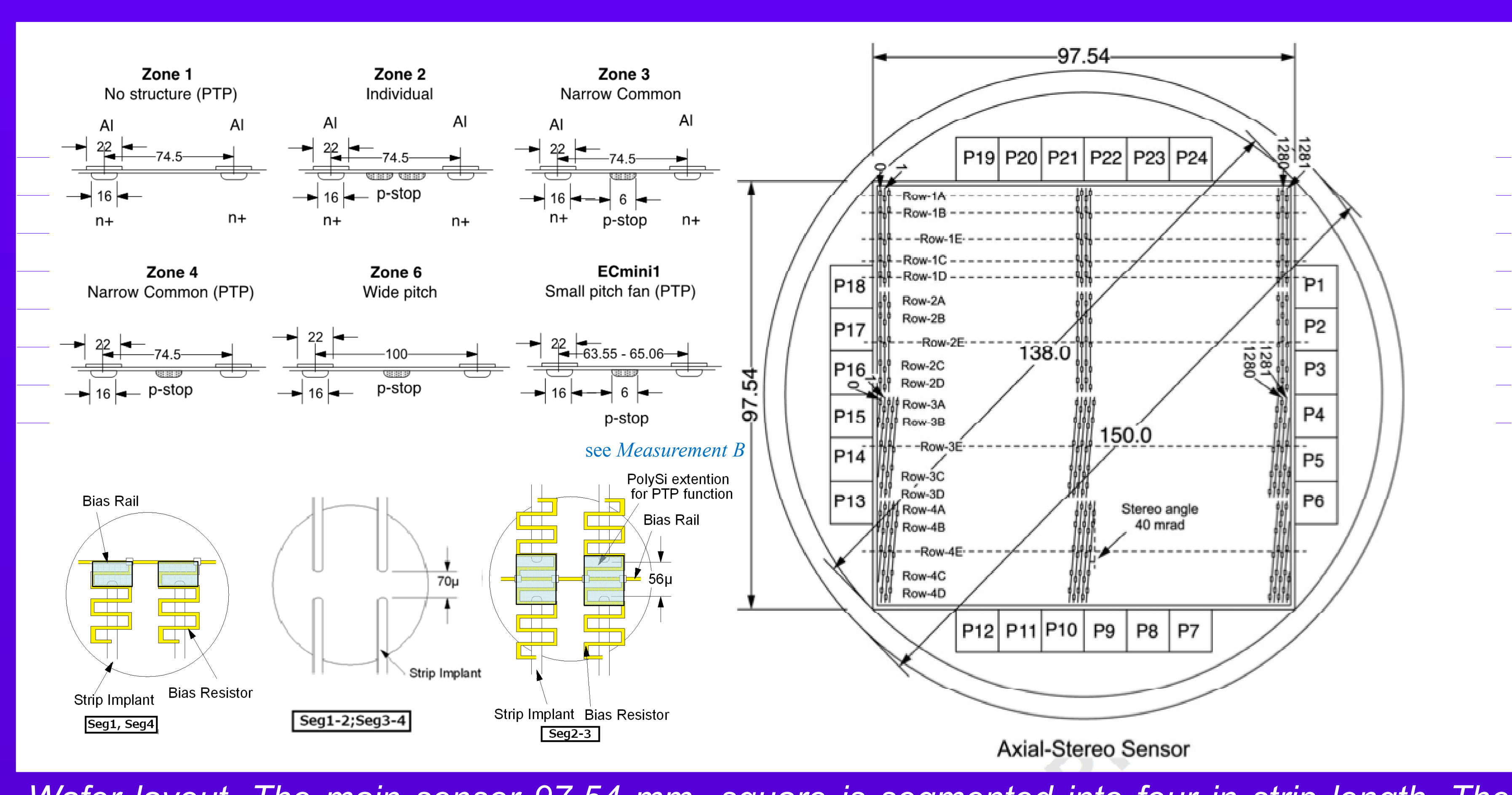

Wafer layout. The main sensor $97.54 \mathrm{~mm}$ square is segmented into four in strip length. The
strips are biased from one ends through poly Si resistors; the Seg2 and Seg3 strips share strips are biased from one ends through poly Si resistors; the Seg2 and Seg3 strips share a common bias rail. The strips in lower two segments are inclined by $40 \mathrm{mrad}$. Miniature examined. The main sensor is a la Z4 where PTP is realized by extending the implant ends closer $(20 \mu \mathrm{m})$ to the bias rail.
Most of the above requirements have been already met The fully irradiated sensors provide a signal of $7.5 \mathrm{ke}^{-}$to $12.5 \mathrm{ke}^{-}$at $500 \mathrm{~V}$ for various irradiation sources. Since the electronics noise is found to be less than $700 \mathrm{e}^{-}$, the signalto-noise ratio will remain above 10 . We continue to monitor such performance in this new prototyping, while accumulating more experience on the sensor fabrication.

Apart from precise values are given in the specifications two modifications should be noted here since the last prototyping ATLAS07.

- $2^{\text {nd }}$ scribe line: provides the possibility to narrow the sensor width. The corresponding measurement is given in Measurement A).

- PTP structure: The ends of the implants are covered fully by poly-Si tied to the bias rails. This helps stabilize the function of punch-through protection, as shown in Measurement B).

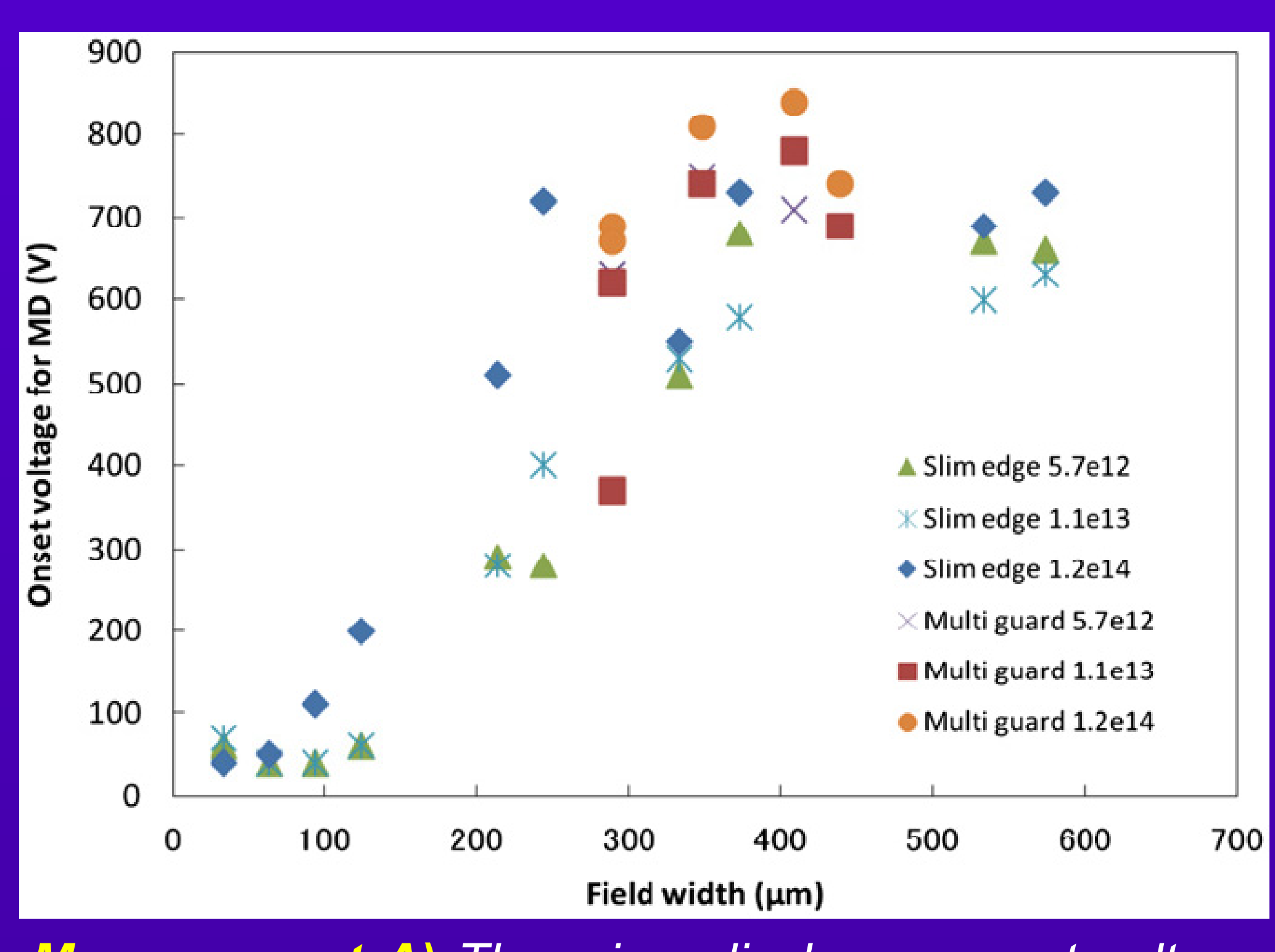

Measurement A) The micro-discharge onset voltage is plotted as a function of the distance between the bias-ring to the scribed edge, after irradiation. A distance of $400 \mu \mathrm{m}$ seems possible. Samples: test
structures of $1 \mathrm{~cm}$ square. The $2^{\text {nd }}$ scribe line at 450 $\mu \mathrm{m}$ in width is from this study.
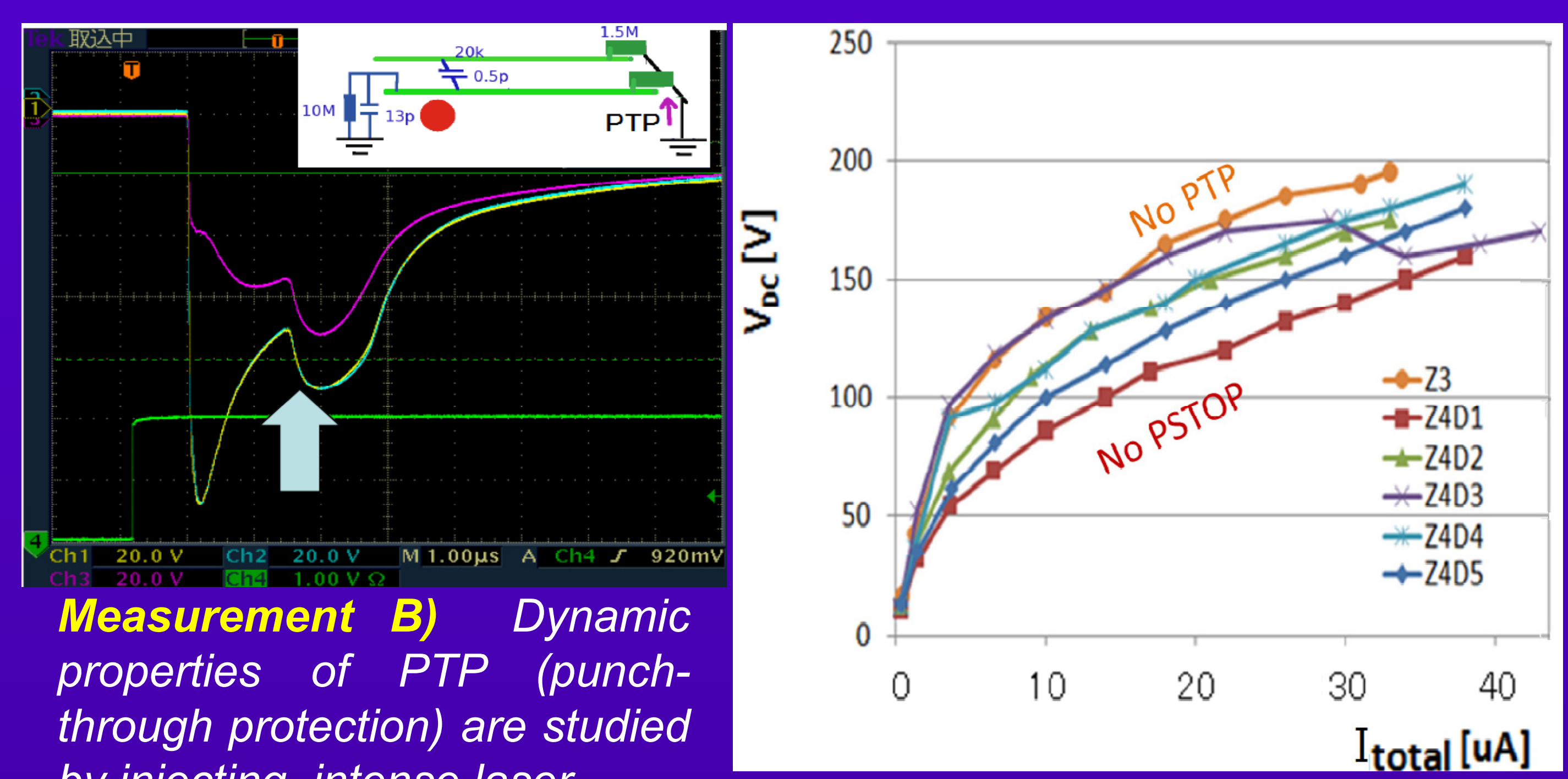

by injecting intense laser.

The implant voltage while PTP is on (up to the arrow shown in the traces. After this point, the impedance changes to increase the implant voltage) is dependent on the laser injection position. For the worst case, i.e. laser (hignal read out from the other end of the PTP structure, the maximum voltages are plotted as function of generated curren

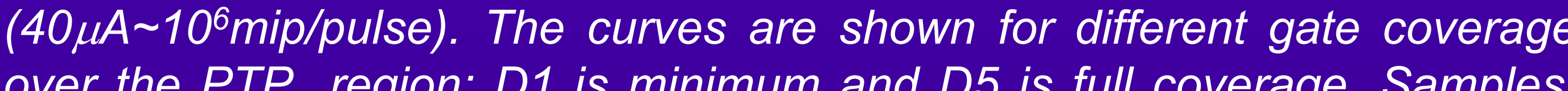
over the PTP region; D1 is minimum and D5 is full coverage. Samples:
test structures of $1 \mathrm{~cm}$ square.

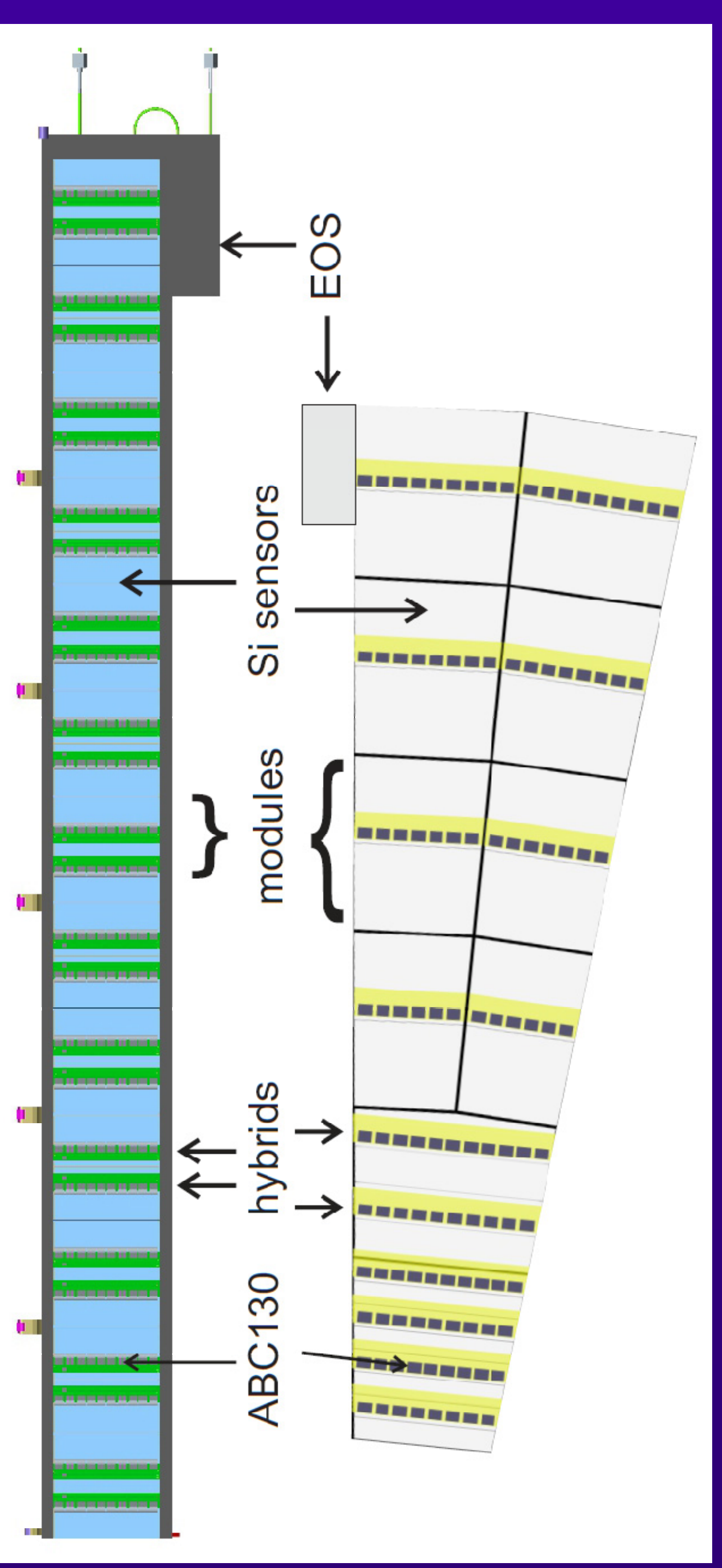

Module Design: The barrel part consists of 512 objects (LS) as drawn. Each LS has 13 silicon sensors on each side providing stereo space point reconstruction, hybrids equipped with ABC130 ASICs, and a common interface (EOS) to the outside. For 256 channels per ABC130, 10 chips are lined on a hybrid, and two hybrids are required for each wafer of short strips. For long strip wafers, one hybrid is equipped per wafer. For the end-caps, the technologies are identical but the shapes are trapezoidal having shortest strips of $2.3 \mathrm{~cm}$ and longest of $6 \mathrm{~cm}$ with the strip pitch varying from 60 to $110 \mu \mathrm{m}$. As drawn there are six different silicon sensor types

Two module designs (STAVE and SUPER MODULE) have demonstrated successfully their mechanical and electrical capabilities with prototype components (e.g. ABC250)

STAVE (baseline design) : Wafers and bus cables underneath are glued on a light structure with integrated cooling (see drawing below). The bus cables provide ASIC shown in the prototype EOS (end

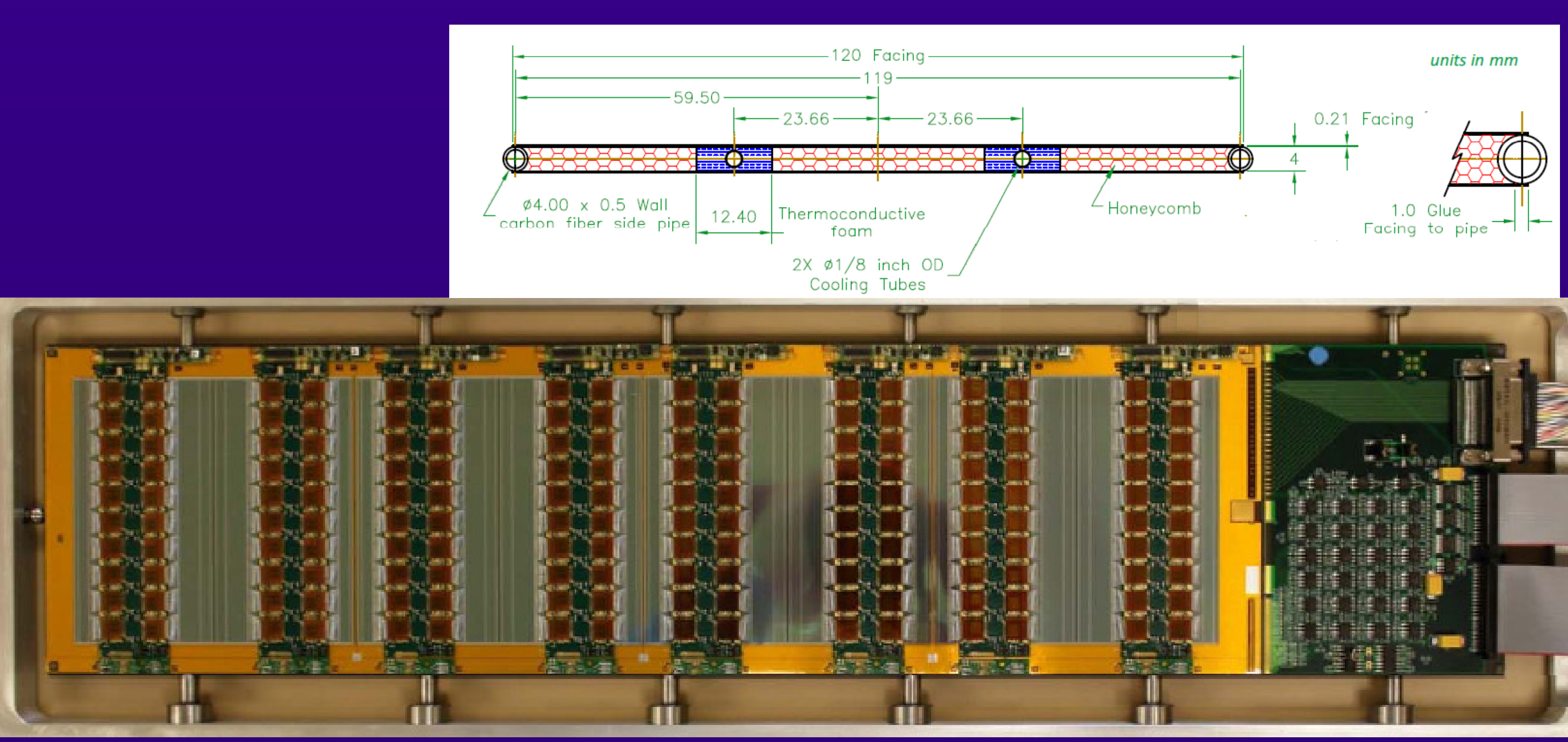

SUPER MODULE (optional design) : Two wafers are glued on (t) which the hybrids are glued bridging over the wafers. 13 such modules are integrated into a supper-module using a light weight frame. The communication is provide through a bus cable running
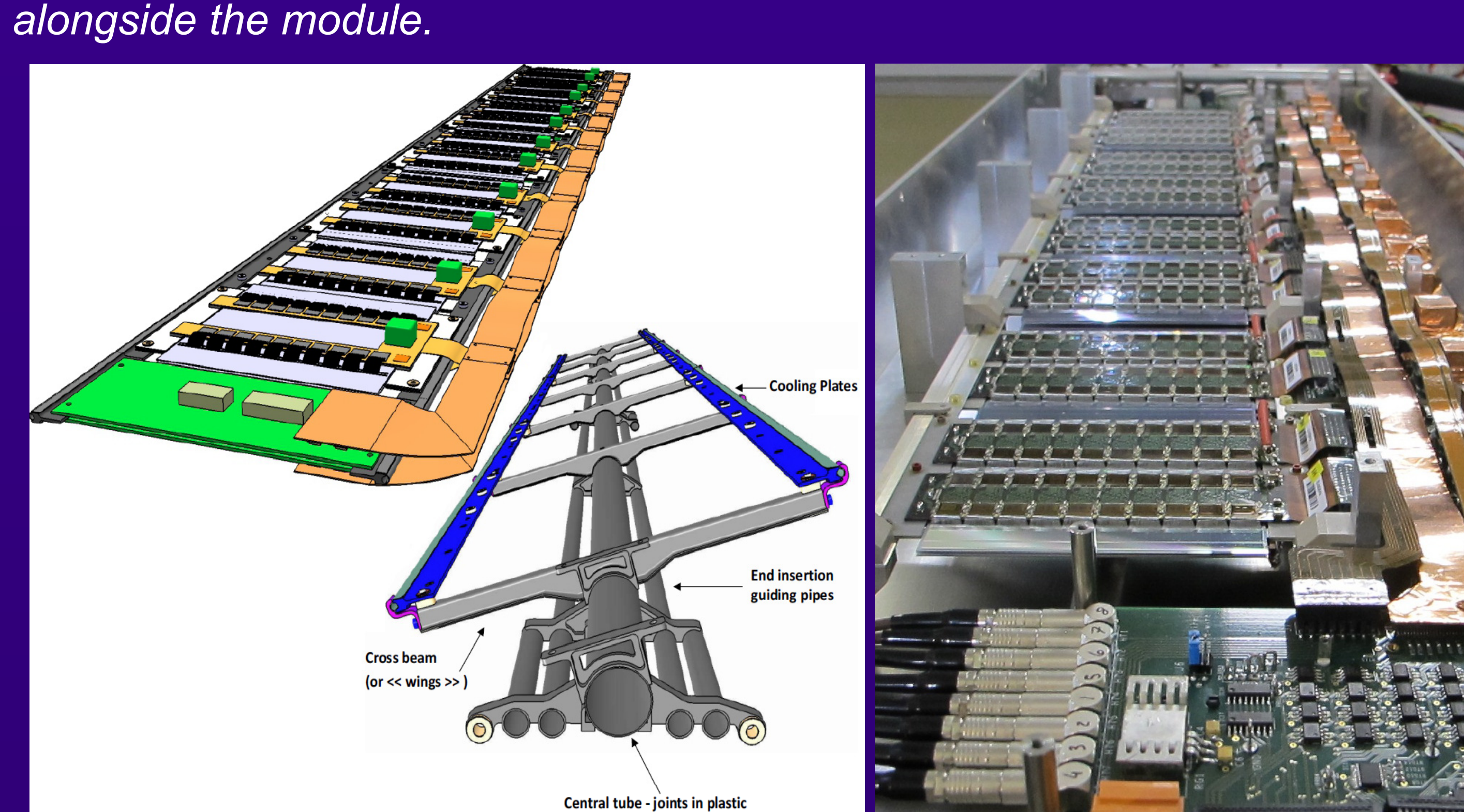